\title{
Le récit: un révélateur des arrière-plans et sous-entendus des métiers, un outil pour former et transformer
}

\author{
$O$ relato: um revelador dos panos de fundo e subentendidos
} do trabalho: uma ferramenta para formar e transformar

Narrative: revealing the background and implications of the work: a tool to develop and transform

Daniel FAïTA (Universidade Aix-Marseille)

\section{RESUMO}

Trabalhos oriundos de diferentes correntes de pensamento, ou mesmo de disciplinas diferentes, como as ciências da educação e a ergonomia, convergem atualmente para um objeto aparentemente comum: fazer com que relatos e discursos de profissionais contribuam para a formação de trabalhadores iniciantes. Se a problemática, de maneira geral, não é nova, sua atualização nas esferas particulares e a utilização concreta de procedimentos dela decorrentes suscitam questões inéditas, ao menos no que diz respeito às ferramentas propostas. Examinaremo-nas fazendo referência a dois exemplos franceses de ações de formação profissional: a autoformação de professores iniciantes por meio da plataforma Néop@ ss-action e a utilização de relatos profissionais na formação à profissão de ergonomista. Assistimos nos dois casos a tentativas de didatizar objetos e processos escolhidos na esfera da produção discursiva.

Palavras-chave: relato; relato profissional; práticas discursivas; autoconfrontação; didatização. 


\section{ABSTRACT}

Work from different currents of thought, and even different disciplines themselves, such as science education and ergonomics are converging towards a seemingly common object: to involve narratives and speeches of professionals in training novice practitioners. While the problem, in general, is not new, its actualization in specific areas and its concrete implementation steps raise new questions, at least on the proposed tools. We will consider them with reference to two examples of French professional development actions: self-development of novice teachers through the platform Neop@ss-action, and the use of narratives in the professional development of ergonomists. We see in both cases attempts to "didactise" objects and processes selected within the sphere of discursive production.

Key-words: Narrative; Professional Narrative; Discursive Practices; Self-Confrontation; "Didactisation".

\section{RESUME}

Des travaux émanant de courants de pensée différents, voire de disciplines elles-mêmes différentes, comme les sciences de l'éducation et l'ergonomie, convergent actuellement vers un objet apparemment commun : mettre à contribution les récits et discours de professionnels dans la formation des praticiens débutants. Si la problématique, dans sa généralité, n'est pas nouvelle, son actualisation dans des sphères particulières et la mise en ouvre concrète des démarches qui en découlent suscitent des questions inédites, au moins concernant les outils proposés. On examinera celles-ci en référence à deux exemples français d'actions de formation professionnelle: l'autoformation des professeurs débutants au moyen de la plateforme Néop@ss-action, et l'utilisation de récits professionnels dans la formation au métier d'ergonome. On assiste dans les deux cas à des tentatives de " didactiser » des objets et processus choisis dans la sphère de la production discursive.

Mots-clés: Récit; récit professionnel; pratiques discursives; autoconfrontation; didactisation. 


\section{Préambule}

La généralisation, même relative, de l'option méthodologique consistant à associer les opérateurs et professionnels aux recherches sur leurs activités, a fortement contribué à remettre à l'ordre du jour les problématiques théoriques touchant au récit, à l'histoire de vie, au discours en général que l'on ne distinguera pas formellement ici, ayant pour principe de recouvrir ces notions sous celle d'énoncé au sens de Bakhtine. Sans pour autant s'engager une fois encore dans les débats - bientôt vieux d'un siècle - ayant jalonné l'analyse du discours narratif et son histoire, on s'en tiendra au fait que ce thème familier ressurgisse dans notre sphère d'activité scientifique, l'analyse du travail. Dans l'univers de la formation, particulièrement des adultes, c'est la question de l'efficacité des ces modes de mises en mots qui ressurgit, sous l'angle de la « didactisation » du récit, au sens large, c'est à dire englobant une variété de formes de médiatisation, dont le film. Les questionnements d'aujourd'hui, on ne peut l'éluder, actualisent aussi les préoccupations de ceux et celles qui, comme Anna Rachel Machado, n'ont cessé de penser que la fréquentation du texte et du discours littéraires, sans oublier la poésie, était nécessaire à la compréhension du vécu, et de mettre en pratique cette conviction. Précisons une nouvelle fois qu'on englobe ici la production discursive sous ses diverses formes, lorsqu'elle fait office « d'activité sur l'activité » (Clot et Faïta, 2000).

La lecture, l'écoute du récit, sont indissociables de l'interprétation des événements de notre vie, et l'on peut trouver dans cette conviction la réponse à la question posée par le titre du précieux petit ouvrage de J.Bruner (2002) : « Pourquoi nous racontons-nous des histoires »? On ajoutera précisément que si le récit ne fournit pas toujours de solutions précises aux situations critiques de la vie courante, il permet au moins de ne pas sombrer dans la facilité des « lieux communs» pour leur donner des réponses.

\section{Introduction}

Les questions touchant à l'expérience professionnelle, autant du point de vue de sa constitution que des moyens d'y accéder, ne sont 
pas nouvelles. Il s'agit d'un objet de réflexion présent dans plusieurs cadres disciplinaires, notamment la psychologie du travail, l'ergonomie et les sciences de l'éducation, sans que le concours des sciences du langage ait à ce jour pesé d'un poids notable. L'intérêt porté à cette problématique est généralement circonscrit dans la volonté d'identifier les éléments de la "compétence ", les sources de "l'efficacité » manifestée par la personne dans son travail. Au second degré, c'est à dire lorsque l'objectif de la recherche n'est pas limité à la volonté de décrire et comprendre l'expérience professionnelle dans ses composantes et ses significations, celles-ci peuvent faire l'objet d'un transfert à des formateurs susceptibles d'en assurer l'acquisition par des débutants ou apprenants. Les récits de l'expérience sont dans ce cas sollicités et recueillis auprès de personnes singulières, considérées dans leur face à face avec la tâche à accomplir, mais sans que soit véritablement prise en compte une relation organique du sujet agissant avec la dimension collective du travail. Ces personnes sont censées détenir des connaissances non triviales, de haut niveau et inaccessibles à la réduction sous forme de simples règles ou routines pour l'action. La recherche se donne alors pour but de révéler et conceptualiser ces connaissances, au profit des transferts mentionnés ci-dessus. Proposant une approche située au carrefour des sciences de l'action énumérées, mais fondée sur le parti pris d'aborder les questions sous l'angle de la dimension langagière de l'activité, on voudrait se démarquer d'un tel positionnement. Suivant nos convictions, acquises au terme de nombreuses années consacrées à l'analyse du travail, et particulièrement du travail enseignant, l'expérience professionnelle demeure un « objet énigmatique ", mieux définissable comme objet d'investigations pluridisciplinaires et collectives visant à sa construction qu'à on ne sait quel gisement de savoir-faire et savoirs en actes en instance de mise au jour (voir à ce sujet Amigues, Faïta et Saujat, 2004). Une certitude toutefois : tout comme l'on pense ici qu'il est vain de prétendre rendre justice au travail humain, en tant qu'objet d'analyse, sans rechercher la proximité maximale de ceux qui l'accomplissent, dans les situations concrètes de leur activité, aucune approche de l'expérience professionnelle ne saurait se concevoir hors de la contribution de ceux qui l'élaborent au fil de leur activité. C'est pourquoi l'on a choisi de traiter des rapports du récit et de l'expérience, ou plus exactement de l'intimité indissoluble de l'un et de l'autre, qui pose tant de problèmes 
à qui envisage de mettre à contribution cet ensemble composite dans la formation professionnelle.

C'est à préciser cette orientation, ainsi qu'à la discussion de deux types d'hypothèses de travail, faisant appel au récit et à la mise en discours, que l'on a consacré les lignes qui suivent.

\section{Récit, mise en discours, et formation. Quelques hypothèses}

On a choisi de centrer cette contribution sur une tendance plus récente, ou du moins en cours de développement rapide dans le domaine de la formation professionnelle. Elle consiste - de façon renouvelée - à mettre l'expérience d'experts ou d'opérateurs confirmés à la disposition des débutants ou des personnes en situation d'apprentissage, sous la forme de dispositifs « d'autoformation ». Dans d'autres cas, l'option retenue consiste à soumettre des apprenants ou débutants aux récits produits par des professionnels confirmés, répondant à la sollicitation de relater l'une ou l'autre de leurs expériences les plus significatives. On ne prétendra pas y voir, dans l'absolu, l'expression de la nouveauté, mais il semble utile d'interroger les tendances à l'œuvre derrière ces développements récents.

Une constante remarquable caractérise ces démarches les plus novatrices, héritières en cela de l'ergonomie dite " de langue française $»^{1}$ : il s'agit de " donner la parole au travail » par la bouche de ceux qui l'accomplissent, enseignants ou travailleurs, pour mettre en circulation cette dimension perceptible, et normalement interprétable de l'activité des personnes concernées, que constitue la relation qu'ils en font. Récits et mises en discours sont en effet indissociables de l'activité professionnelle. S'ils ne participent pas directement de l'action sur l'objet dans tous les domaines d'activité, ils permettent au moins de « faire exister sous forme de discours une totalité qu'on ne maîtrise pas dans le réel », à quoi s'ajoute le fait que tout message « fait sens parce qu'il ne s'en contente pas (François, 1999). Nous

1. On qualifie ainsi le courant issu des travaux et préconisations de Faverge et Ombredane, puis d'A.Wisner, selon lesquels « si on veut comprendre le travail il faut l'observer là où il se déroule et interroger les opérateurs sur ce qui est à faire et comment ils le font ", (Guérin, F.). 
dirions, pour ce qui nous concerne, que le récit rend perceptibles des « totalités » présentes dans le réel mais insaisissables par l'observation parce qu'incommensurables, telles que la majeure partie des dimensions temporelles ou historiques - et spatiales - de toute activité, ce que Clot a exprimé par cette formule notoire : "l'activité ne se touche pas du doigt ». On ajoutera à cela qu'une activité étant toujours « adressée à plusieurs interlocuteurs simultanément, et, elle-même, destinataire de l'activité des autres (...) elle est toujours, en quelque sorte, la réplique à une ou plusieurs autres activités... »(Clot, 1999 : 115). Bakhtine avait déjà formulé à ce propos des idées plus proches de l'objet débattu ici : « ... entre lui (le discours) et celui qui parle, se tapit le milieu vivant, souvent difficile à pénétrer, des discours étrangers sur le même objet, ayant le même thème. C'est dans son interaction vivante avec ce milieu spécifique que le discours peut s'individualiser et s'élaborer stylistiquement » (Bakhtine, 1978 : 100). Le récit comme expression du travail peut donc à la fois intégrer et, concurremment, contredire les autres discours réels ou présumés adressés aux mêmes objets : ceux du « milieu professionnel » au sens large, ceux des savoirs constitués, des normes en vigueur, des - ou de la - technologies, etc.

On fera référence à l'activité enseignante conçue comme travail ${ }^{2}$ pour donner corps à ces hypothèses, dans la mesure où il suffit de référer à la pluralité des " voix » interférant dans la mise en discours de son travail par le professeur, pour brosser un tableau réaliste de la « totalité » spécifique et de la multiplicité des interactions présentes dans celui-ci : voix de la société, traduite par des attentes diversifiées, manifestées par les utilisateurs, de l'institution, représentée par les prescriptions et personnalisée par la hiérarchie, des savoirs à enseigner et de leurs théoriciens, des doctrine didactiques et pédagogiques..., ensemble pesant sur l'expression de l'enseignant et retravaillé par lui dans le concret de son activité. Encore convient-il d'accorder toute sa place à la masse des conditions et des contraintes, en voie de généralisation, mais plus ou moins spécifiques à chaque société : « especificamente no Brasil, os múltiplos papeis que os professores desempenham, o excesso de alunos nas classes, a pouca motivação

2. Sur ces questions, on renverra aux travaux initiés et dirigés par A.R.Machado qui ont actualisé et approfondi au Brésil les directions de recherche représentées en Europe depuis quelques années. 
dos alunos para o ensino escolar, os baixos salários, a multiplicação das horas de trabalho... » (Machado e Magalhães, 2002 : 140, cité par Machado et al., 2011 : 17).

Diverses options méthodologiques, traduisant évidemment des choix théoriques caractérisés, sont matérialisées au service des projets de ce type. Parmi elles, un recours substantiel à l'environnement vidéo numérique semble s'imposer le plus fréquemment, généralement fondé sur la mise en discours de l'expérience professionnelle. Ces discours, enregistrés en réaction au film de situations de travail (particulièrement d'enseignement, en ce qui concerne la présente contribution), mettant en scène des séquences de l'activité professionnelle de sujets agissants auxquelles les intéressés sont ensuite confrontés, ainsi que des opérateurs expérimentés commentant les actes de leurs collègues plus jeunes puis, successivement des « experts », chercheurs ou formateurs. C'est le cas, en France, de la « plateforme »néopass@ction ${ }^{3}$ réunissant actuellement deux équipes sur des objectifs complémentaires, l'accompagnement des élèves dans leurs apprentissages et leur travail scolaire et celui de l'aide à la coordination pédagogique entre les différents professionnels. Ajoutons qu'il s'agit bien, dans ce cas, de procédures orientées vers la production de séquences utilisables $a$ posteriori par des professionnels débutants ou en formation, et non de processus supportant des travaux de recherche initiés dans le cadre méthodologique de « l'autoconfrontation » (Clot et Faïta, 2000, Faïta et Vieira, 2003). Dans ce cas, les présupposés de l'équipe responsable du thème ${ }^{4}$ - aider les élèves en difficulté à travailler - sont orientés vers la production de savoirs nouveaux sur les activités concernées, au prix d'un travail de « co-analyse » associant chercheurs et professionnels désireux de constituer leur propre travail en «objet de pensée » (Clot et Faïta, $2000: 8$ ), en l'occurrence d'équipes pédagogiques formulant une demande à cet égard. De tels actes visent à transformer positivement contenus et situations de travail, tout en produisant des connaissances susceptibles d'alimenter le patrimoine des sciences

3. Plateforme en ligne réalisée par l'Institut Pédagogique National. Propose des ressources fondées sur des travaux de recherche prenant pour objet le travail réel des enseignants : films vidéos de situations de classe, commentés et analysés par des enseignants débutants, des enseignants expérimentés et des chercheurs.

4. ERGAPE : Ergonomie de l'Activité des Professionnels de l'Education, laboratoire ADEF, Aix-Marseille Université. 
mobilisées dans l'étude de l'action. Concrètement, la présentation de la plateforme prend la forme suivante, dans le cas précis du thème signalé : présentation d'une séquence vidéo réalisée dans la classe d'un professeur débutant, en proie aux difficultés habituelles de l'entrée dans le métier, accrues par l'exercice en milieu social défavorisé ; confrontation filmée du débutant à ce même film, commentaires de sa part ; expression sur le même document d'un enseignant expérimenté, et enfin d'un chercheur. Chacune de ces interventions, elles-mêmes filmées et enregistrées, constitue une mise en discours puisant dans la capacité du sujet parlant à interagir avec le « milieu vivant (...) des discours étrangers sur le même objet » (Bakhtine, référence cidessus). Chacun de ces sujets, en effet, réfère explicitement ou non à sa culture professionnelle et/ou théorique, à ses conceptions en matière de mise en pratique, à tout ce qui structure enfin sa réaction en termes d'émotivité. En outre l'enseignant débutant, en ce qu'il intervient en première personne pour commenter ses actes, intègre au commentaire des éléments de son vécu, réfère à des événements du passé proche ou moins proche concernant sa formation, ses rapports avec cette même classe, évoque ses intentions, le projet poursuivi, etc. Il s'engage donc dans un récit qui, pour être fragmentaire, présente la plupart des caractéristiques du genre. Le chercheur, quant à lui, argumente, prélève dans la séquence visionnée des faits, actions, événements, qu'il examine et auxquels il donne un statut en fonction de ses références scientifiques. Au terme de cette procédure d'examen, il distingue un certain nombre d'hypothèses offertes à la réflexion de l'utilisateur de la plateforme (au sujet de la conception de celle-ci, voir l'article de Félix et Espinassy, 2012). Il s'agit là, à n'en pas douter, du motif d'un débat complexe mais indispensable, ayant toute chance d'éclairer la thématique abordée : qu'est ce qui, de ces apports diversifiés, peut être partiellement contradictoires, apporte concrètement un supplément de ressources, une aide efficace et substantielle au débutant en formation? De l'expérience professée par l'expert, de la science invoquée à l'appui des remarques du chercheur, des incertitudes et des retours sur soi du débutant, face à une situation réelle, cette « totalité » peu ou pas dicible mais remarquable, et donc de la superposition ou de la complémentarité de ces discours et récits, qu'est-ce qui est de nature à fournir les ressources pour l'action dont l'utilisateur débutant éprouve lui-même le besoin ? On s'avancera quelque peu, en anticipant sur la suite, pour 
émettre l'hypothèse d'un étayage - fût-il problématique - du regard que l'enseignant en formation porte sur lui-même : « si l'expérience des enseignants échappe largement aux formateurs comme aux chercheurs et aux gestionnaires, elle se révèle souvent insaisissable pour ceux-là mêmes qui en sont les acteurs » (Amigues et al., 2004).

\section{Les apports du récit au carrefour des pratiques et de la connaissance}

On en conviendra, la frontière est ténue dans les faits entre les produits de deux démarches objectivement indissociables : l'une destine à l'autoformation des actes traduisant des connaissances scientifiques d'arrière plan, sous la forme d'images et commentaires diversifiés de celles-ci, l'autre s'inscrit dans le déroulement de processus de recherche, dont la visée est de fournir aux collectifs de professionnels les outils nécessaires à la mise en débat des questions cruciales de leur métier. Comme le notent Félix et Espinassy (2012) : « ... les délais n’ont jamais été aussi courts entre recherche et formation, courant ainsi le risque de se priver du temps de recul nécessaire à la compréhension des phénomènes ». Mais dans les faits, la conséquence des choix privilégiant une relation intime entre intervention et recherche, comme ceux que l'on illustre et soutient ici dans le cadre d'une « recherche fondamentale de terrain » (Saujat, 2010: 66) est d'éloigner paradoxalement le chercheur des moyens de divulguer le produit de son travail sur l'objet de la recherche. Situé dans un processus de « recherche -intervention, à travers lequel étudier le travail est synonyme d'intervenir dans le milieu concerné, d'imprimer à celui-ci des transformations plus ou moins sensibles, et pour finir de rendre des comptes aux intéressés » (Faïta et Saujat, 2011 : 45), il renonce à se positionner en surplomb par rapport au milieu qu'il a lui même contribué à structurer (« milieu associé à la recherche ", ou « communauté scientifique élargie », pour reprendre les termes plus anciens d'Oddone). Praticien parmi les praticiens, il se soumet à l'évaluation de ses actes en référrence au traitement effectif des problèmes réels, ce qui ne signifie pas pour autant qu'il renonce à s'adresser à al communauté scientifique. L'une des conséquences majeures de ces parti-pris réside dans l'importance nouvelle et considérable de ce « compte-rendu » au collectif demandeur 
de l'activité de recherche, de ses débouchés et perspectives. Il est clair que le rapport traditionnel de la recherche à ses résultats, dans le cas des sciences et disciplines dites " appliquées » (catégorie limitative que l'on réfutera ici, voir ci-dessous la mise en débat des rapports singularité - généralité), se trouve fondamentalement transformé. Il ne s'agit plus de livrer aux intéressés un produit supposé satisfaire à la commande, mais d'adresser aux demandeurs un discours, des textes, remplissant une double fonction:

- mettre à la disposition des intéressés les éléments d'une compréhension différente de leur vécu professionnel, principalement en réaction à leurs conceptions antérieures, leur offrir de ce fait les moyens nécessaires à la transformation positive - mais non préconçue - des conditions, contenus et modalités de leur activité professionnelle;

- consolider ou aménager les cadres conceptuels et méthodologiques permettant à la recherche elle-même d'anticiper sur les problématiques à venir à partir de l'expérience réalisée, ce qui est normalement porteur de savoirs nouveaux, de par la projection d'hypothèses sur des situations à venir, demandant vérification ou invalidation.

En ce sens, et suivant en cela Saujat (2010:66), on abondera dans le sens de Sensevy: " plus la singularité d'un individu dans l'action a été effectivement atteinte, plus nous pourrons apprendre des ressorts de l'action, en général: non pas parce qu'on se donnera le droit de décréter l'universalité du singulier, mais bien plutôt parce que rendre raison d'une action particulière, c'est se rendre compétent dans l'étude d'une autre, et de beaucoup d'autres » (Sensévy, 1999: 142).

Prenant au mot cette hypothèse, on se proposera de considérer qu'à l'utilité de donner la parole au travail par l'entremise des récits et discours de ceux qui le réalisent, répond celle de considérer les productions discursives de ceux qui font profession d'expertise ou de connaissance scientifique comme la mise en mots de leur activité propre de travail. Si les premiers donnent sens et restructurent, à leur propre usage et pour les besoins de la co-analyse, les éléments de leur expérience, les seconds s'engagent dans une activité homologue dont l'objet est alors constitué par le travail des autres. Chacun demeure 
l'acteur, mais aussi, en dernière analyse, le propriétaire de son propre travail. Cela souligne la validité de la relation entre « singularité » et anticipation, évoquée ci-dessus.

\section{Des discours entrecroisés, une superposition des rapports dialogiques}

Dans tous les cas, on voit clairement comment les situations de travail investiguées dans le cadre de l'intervention ne sont en aucun cas considérées au titre de gisements de données, à traiter hors champ pour renvoyer aux intéressés du pensé ailleurs, supposé les renseigner de l'extérieur sur leur propre vécu. Il s'agit au contraire de points de départ pour des activités convergentes de réflexion, aboutissant à ce que Clot qualifie «d'échange d'activités » (..) dont l'objet ne peut être circonscrit dans l'abstraction ni a priori, mais seulement en termes d'objectifs partiellement communs.

C'est le croisement des ces activités (discours, récits) réciproquement adressées, sur et à partir des situations de travail, qui doit fournir in fine, au terme du processus inscrit dans la durée, des ressources utiles en premier lieu à la mesure de l'écart entre ce que le débutant perçoit de sa propre activité et ce que lui enseigne le regard des autres sur cette activité. «Durant tout ce processus, acteurs et chercheurs sont donc engagés dans un travail de co-construction, des faits et de co-analyse des situations de travail qui, après avoir été jugés par les protagonistes comme emblématiques de leur action, sont remis en circulation auprès d'un collectif élargi » (Félix et Espinassy, 2012). Face aux transformations du métier d'enseignant, en l'occurrence, créatrices de nouveaux besoins des professionnels, ce processus complexe d'échange et de controverse est seul susceptible de fournir des ressources pour l'action par l'entremise de formations conçues à nouveaux frais.

Il est évident, bien que trop peu étudié, que les échanges supportant le processus en question reposent essentiellement sur la production discursive des protagonistes, de leur capacité à mettre en discours leur vécu des situations, à expliciter les choix, les priorités, les renoncements, les ressentis, etc. tissant leur expérience professionnelle, comme leur perception de ce qui dans l'environnement et l'histoire de leur fonction les affecte plus ou moins profondément. 
Du côté des « experts » ou chercheurs, c'est la capacité à formuler et reformuler des hypothèses relatives aux faits recueillis, à leur traitement dans le cadre méthodologique retenu, des mises en discours destinées au collectif associé. Il s'agit donc bien, dans tous les cas, de produire en contrepoint aux activités respectives les éléments de l'échange, de la compréhension réciproque, voire de la controverse et de la conviction. D'un côté comme de l'autre, il s'agit bien de « donner la parole au travail » sous les formes appropriées, comme le préconise l'ergonomie francophone, évoquée ci-dessus.

\section{Le récit « professionnel », artefact ou réalité ?}

D'autres perspectives émergent à l'heure actuelle, parmi lesquelles on retiendra le projet de mettre le récit à contribution, en tant que tel, dans la formation professionnelle des ergonomes. C'est le propos central de la thèse soutenue en 2011 par J.Beaujouan, considérée ici comme une référence. Ce texte a le mérite de parcourir, de manière quasi exhaustive, les concepts et hypothèses permettant de préciser les apports et limites du récit dans la construction des apprentissages, eu égard aux caractéristiques du " métier d'ergonome.. » ainsi qu'à celles des pratiques inscrites dans une "visée d'apprentissage ". " Conceptualisation de l'action », « développement de compétences sociales et relationnelles ", " aide à la résolution de problèmes ", « compréhension du monde », etc., participent parmi d'autres des vertus présupposées du récit à « visée didactique». Faisant l'hypothèse d'un rôle positif du récit professionnel dans cette formation, il s'interroge d'abord sur les propriétés communes et les facteurs de différenciation permettant ou non d'identifier une telle catégorie : « récit professionnel ». Une liste de critères, comme « finalisation du récit », ou encore « succession d'actions intentionnelles » - retenus parmi d'autres possibles - est censée permettre d'identifier les « effets produits sur les étudiants destinataires » (p.120-121), en association avec des marques d'identification (marqueurs langagiers) formellement reconnaissables.

L'intérêt manifeste d'une approche de ce type réside dans la tentative de mettre en regard des caractéristiques concrètes, parfois sous forme de « variables » plus ou moins informelles, telle « l'intention 
didactique exprimée ", et les attentes de la formation, en termes de cibles visées. Sans dériver pour autant vers l'illusion mécaniste d'une corrélation systématique entre caractéristiques professionnelles et impact en formation, l'analyse quantitative proposée évalue positivement l'adéquation de l'outil « récit professionnel 》 aux objectifs de la formation. De ce point de vue, ce dernier constitue bel et bien un artefact, déterminé de bout en bout par le projet didactique, même si de toute évidence les étudiants n'en bénéficient pas tous de la même manière, ce qui en retour, toujours dans la même démarche, autorise à pressentir des « types de récits efficaces », tout au moins plus efficaces que d'autres, ainsi que des " profils d'étudiants réceptifs ». Il est intéressant de tenter une typologie de ces récits, proposés par des professionnels confirmés, proportionnellement à la réceptivité différenciée manifestée par les apprenants. De la même façon, l'enjeu que constitue pour la formation l'étude de la « réflexivité » suscitée par les récits (p.175) s'apprécie à la mesure de la capacité des apprenants à « dialoguer avec leur propre pratique et celle des autres ».

Un tel projet, soutenu par l'idée d'une possible mise en perspective du produit d'une activité narrative, distanciée de l'action, voué à susciter chez autrui des conduites et des réponses entrant dans un cadre prédéfini, semble contradictoire avec les présupposés que l'on revendique ici, à savoir la critique de toute objectivation forcée d'un processus symbolique et/ou langagier afin de le transformer en support de formation jouant de façon univoque. On persiste à partager la conviction de François, selon qui par exemple la prétention du sujet parlant d'affecter à son discours des significations définies, et seulement elles, relève de l'illusion. De même, et dans l'ordre de l'activité « productive » cette fois, on ajoutera en reprenant Wallon (1942-1970) que l'objet de l'activité est toujours constitué par « les manières de s'y prendre dans la relation à autrui par l'entremise de l'action sur l'objet de cette action », ce que Clot (1999: 77), traduit ainsi: « ... l'action de travail (...) ne vaut pas en elle-même. Elle vaut au regard des autres activités du sujet, bien sûr, mais d'abord au regard des activités des autres qui donnent à cette tâche sa contenance symbolique et collective $»$.

Quelle que soit la volonté présidant à la production d'un récit «professionnel » par l'expert, l'objet achevé offre l'apparence d'un 
tout organique, clos sur lui-même et censé offrir à autrui l'expression d'un dessein tendu vers son objectif. Il s'inscrit cependant dans un paradigme particulier, pour éviter de solliciter ici sans plus de réflexion la notion de " genre », celui des artefacts. On entend par là ces objets matérialisant les acquis de ceux des sujets et collectifs de sujets ayant affronté antérieurement les épreuves des apprentissages et les écueils des pratiques, et permettant ainsi au travail de se présenter " grâce aux traces qu'il laisse, objets, signes, outils et règles pour les générations qui se succèdent » (Clot, 1999: 74). Il reste que de tels objets, culturels par nature, sont particulièrement complexes, ainsi que le laisse présager la somme de débats internes, de choix et d'arbitrages, sans oublier enfin les multiples aléas de l'histoire qui accompagnent leur production. S'y ajoutent les difficultés propres à la mise en mots, les choix inévitables entre façons de signifier différentes, et parfois divergentes, les comptes à régler - si possible - avec les déterminants cachés ou éloignés, tels les savoirs, les expériences éloignées dans le temps, les normes sociales...

Objectiver l'efficacité du récit en tant qu'instrument de médiation et de formation constitue donc une tâche difficile. Il convient sans doute, dans cette entreprise, d'évaluer dans la mesure du possible les effets associés à telles ou telles caractéristiques, en corrélation avec les dispositions différenciées des apprenants. Mais il est non moins important de questionner le mode de production des discours narratifs, les préoccupations propres aux narrateurs ainsi que la façon dont ceuxci se confrontent aux vicissitudes de l'énonciation.

\section{Les méandres du discours face aux servitudes de sa « didactisation »}

Le récit, le discours saisi en tant qu'objet fini, on l'a avancé, ne présente que l'apparence de l'achèvement. Les raisons en sont multiples, mais les principales peuvent ressortir à deux catégories : d'abord le fait que le récit disponible à l'état d'objet, produit de l'activité du narrateur, représente avant tout un moment d'équilibre entre le dernier stade en date de cette activité avant sa reprise et sa transformation, d'une manière ou d'une autre, dans le rapport dialogique dont il participe. On se réfèrera, à ce sujet, au rapport complexe pressenti par le texte 
de J.Beaujouan entre types de récits et « profils réceptifs d'étudiants ». S'il ne fait pas de doute que les effets escomptés dépendent avant tout de la rencontre entre le récit - auquel on persistera à substituer le terme d' «énoncé »- proposé et l'activité en retour de son destinataire, cette « compréhension responsive active » familière aux lecteurs de Bakhtine, il ne fait alors aucun doute que le moment de la rencontre entre les deux protagonistes du rapport n'est qu'un épisode parmi d'autres du processus engagé, dont le terme demeure inconnu.

Mais d'autre part, il n'est pas assuré, loin s'en faut, que la substance de ces activités réciproquement orientées se résume au matériau langagier enclos entre les limites du discours ou du texte. Il est fort probable qu'en termes de sens les choix de l'énonciateur comptent autant que les significations résultant de ceux-ci. Reste à savoir comment ils se manifestent, mais si « les rapports dialogiques sont impossibles sans les rapports logiques et sémantiques (...) ils ne peuvent s'y réduire, pas plus qu'aux rapports de signification objective dépourvus de mouvement dialogique (...). Pour devenir dialogiques, ces derniers doivent s'incarner (...) recevoir un auteur... c'est à dire qu'un sujet de l'énoncé y exprime sa position » (Bakhtine....). c'est donc probablement à cette « position » manifestée, décelée ou devinée, qu'est également redevable la dynamique du dialogue, ici le travail réciproque de l'expert et de l'apprenant, autant qu'aux catégories verbales et structurelles choisies. Le rapport du discours tenu à son objet déclaré n'est donc pas aussi transparent, et comme l'affirme fréquemment François, c'est dans le discours qu'apparaît l'impossibilité de dire les choses, ce qui signale comment ce dernier peut aussi produire du sens par défaut, dans des rapports ne se limitant pas à la mise en relation des mots et des choses mais englobant, parfois de façon contradictoire les faits, les événements plus ou moins identifiables supportant cette relation. S'il demeure certain que l'objet du récit détermine grandement celui-ci, car structurant le dialogue (Nonnon, 1996), ce n'est sans doute pas de manière constante et inaltérable, la façon dont on parle des choses pouvant se substituer, précisément en tant qu'objet, à ce dont on parle. A l'exclusion des " genres scolaires bien typés, au service d'apprentissages » (François, 1990: 70), beaucoup dépend en effet des mouvements affectant le rapport entre le discours, le contexte qu'il se donne, la façon dont le locuteur renvoie à celui-ci, en découpe les éléments, dont il propose des équivalences ou des oppositions entre 
ceux-ci, et pour finir produit ou non de l'inattendu, du dérangeant, du contradictoire... François (ibidem) se posait la question de savoir si l'on apprenait en discutant, ce à quoi l'on ajoutera la question suivante : n'apprend on que ce que l'on est censé apprendre en écoutant un récit ou une histoire?

Bruner insiste sur l'existence de deux modes de pensée, chacun représentant « une façon particulière d'ordonner l'expérience, de construire la réalité » (Bruner, 2000: 27). Il ajoute : " une bonne histoire et une argumentation bien menée appartiennent à des genres naturels différents. Toutes deux peuvent être utilisées pour convaincre » (Bruner, ibidem). L 'essentiel réside alors dans le fait que les critères permettant de juger ou évaluer l'une et l'autre sont de nature différente. On ajoutera que ceux-ci n'interviennent pas nécessairement de manière séparée, ne sont pas forcément exclusifs les uns des autres dans une même unité de narration, dans laquelle peuvent coexister des traits exprimant ces «modes » divergents. Cela permet d'entrevoir des contradictions internes au discours produisant de possibles effets de sens. Bruner encore, suggère que « le récit, quelle qu'en soit la forme, consiste en une dialectique entre ce que nous attendons et ce qui se produit effectivement », ajoutant «l'histoire est extrêmement sensible à tout ce qui contrarie notre sens du 'normal'» (Bruner, 2002: 27). Par conséquent, un lien se dessine entre le récit produit pour instruire, convaincre, argumenté à ces fins, et l'histoire, réellement présente dans ce récit lui-même mais pas sciemment projetée, qui jouera la fonction contradictoire de nous surprendre. La différence entre ce lien et un terrain véritablement commun peut s'annuler, si précisément l'intentionnalité didactique du producteur de discours a pour effet de placer son auditeur devant l'écart inattendu entre ce qu'il croyait savoir ou maîtriser, éventuellement de lui-même, de ses propres connaissances ou compétences, et ce que lui signale le discours de l'autre. La révélation de la distance entre ce que l'on croit savoir, ou ce que l'on croit juste, ou vrai, et ce que donne à percevoir de différent le discours d'autrui entre à n'en pas douter dans le processus de transformation impliqué par la formation. Celui-ci peut sans aucun doute se nourrir des façons dont le récit formateur produit des effets imprévus, tributaires plus de sa genèse, des intentions d'arrière plan et des contraintes structurantes que de la visée proprement didactique. 
On tient pour établi qu'au principe du récit figurent à la fois « sa capacité à exprimer les idées confinées dans les conventions du quotidien (ce qu'il convient de penser et dire) » (Bruner, 2002: 34) ainsi qu'à « donner forme à notre expérience quotidienne » grâce aux modèles narratifs de la réalité. Citant fort à propos Ricoeur (1983), selon qui rien n'entre dans l'expérience qui ne soit raconté, J.Beaujouan note que « raconter son expérience par le récit est un moyen de ressaisir le temps et de transformer ce rapport en identité narrative » (Beaujouan : 70). Les sujets ayant vécu l'épisode fourmillant d'événements plus ou moins désarticulés peuvent reconstruire les épisodes dans un enchaînement intelligible à partir de l'intrigue qui s'accroche sur la fin de l'histoire et dont ils ont désormais la connaissance. Ils se trouvent ainsi « libérés de la préoccupation de l'agir », et « il n'est pas étonnant que les opérateurs doivent passer par le moment du récit pour construire les relations de signification qui leur permettront de maîtriser leur système » (Pastré: 33). Dans l'optique d'un développement de la «réflexivité» des apprenants sur leurs propres actions, il est donc logique d'envisager le recours au récit en formation comme support à l'examen et la compréhension des échecs et réussites de leurs actes. Nuance capitale, la contribution du récit aux apprentissages n'est pas uniquement consécutive à ses qualités et propriétés, « mais surtout à la manière dont ses destinataires vont le percevoir, l'interpréter, l'exploiter » (Beaujouan: 97). Il n'est donc pas question de préjuger de la forme ni de la portée de cette exploitation, pas plus que de sous-estimer les difficultés objectives de verbalisation entravant le processus, depuis la mise en mots - et donc la reconstruction - de l'expérience professionnelle jusqu'à la mise en cohérence des éléments du récit. C'est à ce stade de la réflexion que de possibles divergences d'analyse méritent d'être évoquées.

\section{Retour sur le rapport du récit à ses objets, du destinataire présumé à sa place dans le dialogue}

Un autre sujet de préoccupation s'impose si l'on poursuit, avec Bruner, l'exploration des ressemblances et divergences réunissant et opposant les « deux modes de pensée » examinés plus haut. Il semble évident que le récit professionnel à visée didactique, outillé comme on l'a supposé afin de remettre en cohérence les « événements disparates » 
jalonnant le vécu, participe de la catégorie «paradigmatique » (Bruner, 2000: 28-29), c'est à dire propose « des outils grâce auxquels des propositions de portée générale peuvent être extraites de constatations liées à des contextes particuliers » (ibidem), en appui sur l'enchainement logique de catégories référées à des éléments vérifiables. Il ne paraît pas vraisemblable, à l'opposé, que de tels énoncés cherchent à « donner du sens à l'expérience » au moyen de ressources puisées dans un mode narratif qui sacrifierait la vérité empirique à une crédibilité de nature dramatique. Une telle fonction reste l'apanage de celui qui fait preuve d'imagination dans le mode narratif, produisant de 'belles histoires, des drames poignants', etc. (ibidem: 29). L'opposition entre logique, vérité, et d'autre part imaginaire n'est elle pas cependant plus ténue qu'il y paraît ? Certainement pas si l'on s'en tient aux critères classiques distinguant formellement un mode de l'autre, le doute étant cependant permis si l'on se place du point de vue de la production du récit en tant qu'activité orientée vers l'apprentissage par autrui des règles et principes d'un métier. Dans la démarche étudiée ici, les récits professionnels identifiés comme tels ont été suivis d'entretiens auprès des narrateurs concernés, visant à comprendre les choix ayant motivé leur production devant les ergonomes en formation, les raisons justifiant les manières de procéder, comme l'insistance sur certains points, le choix ou non d'utiliser un support, etc. (Beaujouan: 121). Or, il est édifiant de constater que chez plusieurs des professionnels sollicités, si le discours produit en réponse à la consigne commence par la justification d'un dessein intrinsèquement didactique rendre perceptible une réalité complexe au moyen d'un discours adapté à la « mise en ordre » des éléments du réel - il évolue assez rapidement vers des choix narratifs, voire vers des formes de dramatisation :

Intervenant $\mathrm{X}$ - C'est cette diversité là qui est le fil conducteur de l'intervention. Alors après il y avait certaines expériences qui sont beaucoup plus loin dans ma mémoire que d'autres... (...) En fait j'ai fait le choix de raconter certaines partie d'intervention, pas tout...

- J'ai l'impression quej'ai moins raconté le temps vécu, que j'ai plus pris de temps que pour raconter l'exemple en tant que tel de chacun des projets. J'ai plus développé les principes, plus raconté ce qui s'est passé dans le sens descriptif. 
Intervenant $\mathrm{Y}$ - Expliquer à quelqu'un, par des mots, ce qu'est notre boulot, le travail tout simplement, c'est pas si simple. Et donc, du coup, il faut rester me semble-t-il super simple, concret et dans le contenu, dans l'objectif aussi, ce quej'ai raconté c'était pour illustrer, parler de notre travail... (...) L'objectif c'était vraiment de faire partager une expérience de travail, et pour ça ben... les histoires, et l'histoire que j'ai racontée... ben je vois pas comment faire différemment...

- Alors j'ai choisi cet exemple (...) que j'ai fait vivre. Je gesticulais à côté du plan et je voulais que ce soit visuel...!

- La grosse difficulté c'est que c'est une histoire (...), qui était extrêmement complexe et doncj'ai essayé de réaliser quelques simplifications extrêmes (...) l'idée c'est d'essayer de sortir de tout ça quelque chose qui est complètement subjectif, au final...

- Par exemple, y a plein de détails sur comment a mûri le projet... comment est née cette idée là de placer la passerelle ici... J'ai raconté que c'est l'architecte qui m'a fait trois propositions... C'est pas vrai! C'est pas l'architecte...

(Beaujouan, op. cité, vol. d'annexes p. 43-44-45-51-52-53).

Il est clair que la conduite du récit obéit initialement à la visée didactique préconçue: simplifier, rendre accessible et perceptible, et pour cela ordonner les références, sélectionner parmi les événements ceux qui paraissent de nature à servir le projet. Assez vite cependant, d'autres contraintes se manifestent, comme l'impossibilité de réduire le temps de la narration au temps effectif de l'action, ou l'inverse : voir ci-dessus la référence à François, affirmant que la mise en récit révèle l'impossibilité de dire certaines choses.

Le paradoxe développé par l'intervenant $\mathrm{Y}$ est de taille, à ce propos: la complexité des situations réelles exigerait, dans une mise en récit « efficace », une simplification inversement proportionnelle, au point - c'est important - de dissocier le récit de son objet initial (l'activité professionnelle, les actes, les objets réels) pour s'engager dans « l'illustration » par les histoires, de manière à faire partager une expérience (souligné par nous), au prix éventuel de " gesticulations » convoquant le « visuel », c'est à dire s'adressant à la capacité présumée 
des apprenants de s'impliquer physiquement, sensoriellement, dans l'histoire... Et pour terminer, on notera la torsion infligée en toute conscience à la réalité historique - bel exemple du sacrifice de la vérité au profit de la crédibilité de l'argument - lorsque l'histoire et celui qui la raconte travestissent les faits.

En poursuivant les pistes envisagées ci-dessus, la divergence présumée du récit «paradigmatique » et de l'histoire dramatisée paraît alors beaucoup plus floue. On s'autorisera à reconsidérer l'hypothèse de Bakhtine en examinant de plus près une dimension du récit « à visée didactique " dans laquelle le modèle subjectif du destinataire et de ses attentes (voir à ce sujet Bakhtine, 1984: 303-304), préside à la «thématisation » caractéristique de l'énoncé. En d'autres termes, comment la conception de la tâche qu'il accomplit, en fonction des consignes explicitées et des attentes supposées de l'interlocuteur, induit la production du récit par le professionnel sous une forme spécifique.

On proposera alors de réviser, au moins partiellement, l'idée suivant laquelle l' « efficacité » du récit en formation dépendrait avant tout de la capacité du narrateur à ordonner logiquement les matériaux « disparates » jonchant les situations réelles. On se demandera si le plus important en matière, toujours, d'efficacité, n'est pas d'induire l'apprenant en tentation, tentation de mesurer en quoi et comment dans les situations concrètes affrontées le narrateur a procédé à des choix, a pris des options conformes à ce que lui-même aurait envisagé de faire, ou au contraire contradictoires.

\section{Conclusion}

Récit professionnel et plateforme d'autoformation ont ceci de commun qu'ils offrent à l'apprenant des objets à construire, selon des modalités partiellement différentes. A la base de ce processus, réside probablement un rapport mouvant entre l'intention (ou la « préoccupation », pour reprendre la notion en usage en psychologie du travail) et l'action des professionnels, experts ou chercheurs. Mais selon toute probabilité un rapport tel qu'il ne se confine pas dans le débat intérieur entre le dessein discursif du sujet agissant, son intention de signifier, et les obstacles qui s'y opposent. Un rapport qui au contraire 
se montre par les événements et accidents du discours et manifeste ce qui est difficile ou impossible à dire. Dans le cas de la plateforme, les interventions des protagonistes expérimentés et chercheurs s'organisent moins autour de l'objet concret, le film de la situation de classe de l'enseignant novice, que de l'objet abstrait et néanmoins traditionnel de leurs activités respectives: les enseignants chevronnés peuvent par exemple centrer leur discours sur ce qui à leurs yeux «ne marche pas » dans les actes des débutants, par rapport à l'expérience professionnelle communément partagée, ainsi qu'à la norme pédagogique, alors que les chercheurs vont évaluer la même prestation, principalement certains aspects de celle-ci, en fonction d'outils critiques produits dans le cadre de savoirs scientifiquement élaborés. Ces derniers, rapportés à la singularité des actes examinés, ne peuvent que difficilement intégrer « le développement de l'activité de l'enseignant débutant», qui « se fraie (...) un chemin à travers des conflits vitaux » (Saujat, 2011). Les activités des uns et des autres s'orientent vers des objets, répondent à des objectifs, qui ni dans l'un ni dans l'autre cas ne cadrent avec ceux de l'enseignant concrètement aux prises avec une situation donnée.

On a pu voir que les « récits professionnels» des ergonomes, structurés par leurs auteurs conformément à leur « visée didactique ", s'organisent probablement en fonction d'impératifs énonciatifs beaucoup plus variés, faisant appel à une mixité sémiologique peu prévisible.

Dans tous les cas, l'hypothèse d'une " efficacité » recherchée, ou présumée, de ces actes, n'est pas en cause, dans la mesure où pour reprendre encore l'affirmation de François, c'est l'écart constaté par le débutant avec ses propres conceptions et actions qui induit une démarche productive, contribuant à un processus de développement. Dans les deux cas, le pouvoir heuristique consiste à faire surgir une variété de lectures possibles des conduites effectives et envisageables par l'apprenant.

De ce point de vue, récit et discours fonctionnent comme introductions à l'action, non comme exposés significatifs des pratiques à reproduire, et confirment les potentialités qu'ils recèlent de ce point de vue. 
Recebido em março de 2013

Aprovado em abril de 2015

E-mail:

d.fait@wanadoo.fr

\section{Blibliographie}

Amigues, R., Faïta, D. \& Saujat, F. 2004. L'autoconfrontation croisée: une méthode pour analyser l'activité enseignante et susciter le développement de l'expérience professionnelle. Bulletin de psychologie, 469, 41-44.

BaKhtine, M. 1978. Esthétique et théorie du roman, Paris, Gallimard. . 1984. Esthétique de la création verbale. Paris, Gallimard.

Beaujouan, J. 2011. Contribution des récits professionnels à l'apprentisssage d'un métier. Le cas d'une formation d'ergonome. Thèse pour le doctorat de l'Université Bordeaux Segalen. Mention: Ergonomie.

Bruner, J. 2000. Culture et modes de pensée. Paris Retz. 2002. Pourquoi nous racontons-nous des histoires? Paris, Retz.

CLOT, Y. 1999. La fonction psychologique du travail. Paris. P.U.F.

Clot, Y. et Faïta, D. 2000. " Genres et styles en analyse du travail ». Travailler, $\mathrm{n}^{\circ} 4: 7-43$.

FaÏta, D. et Vieira, M. 2003. "Réflexions méthodologiques sur l'autoconfrontation croisée ». SKHOLÊ Hors Série I : 57-69.

Faïta, D. et Saujat, F. 2011. « Développer l'activité des enseignants pour comprendre et transformer leur travail : un cadre théorique et méthodologique ». In Yvon, F. et Saussez, F. Des outils méthodologiques et théoriques pour l'intervention et la formation. Presses de l'Université de Laval.

FÉLIX et EsPINASSY. 2012. « De l'intervention-recherche à la production de ressources : quelle didactisation de l'activité pour la formation des enseignants ? ». Recherche et formation.

François, F. 1990. La communication inégale, Neuchâtel, Delachaux et Niestlé.

. 1999. « Mot et dialogue chez Vygotski et Bakhtine. In Clot, Y. (éd.), Avec Vygotski : 189-206. Paris, La Dispute.

GUÉRIN, F. et al. 1991. Comprendre kle travail pour le transformer. Montrouge, Editions de l'ANACT.

Machado, A.R. (org.). 2004. O ensino como trabalho. Londrina, EDUEL. 
Machado A.R.; Gouvea Lousada, E.; D’Orange Ferreira, E. (org.). 2011. O Professor e seu trabalho. Campinas, Mercado Letras.

Nonnon, E. 1996. "Activités argumentatives et élaboration de connaissances nouvelles : le dialogue comme espace d'exploration », Langue française, $\mathrm{n}^{\circ} 112$ : 67-87.

PASTRÉ, P. 1999. « La conceptualisation dans l'action. Bilan et nouvelles perspectives ». Education Permanente, (139), 17-35.

Ricoeur, P. 1983. Temps et récit. Tome 1 : l'intrigue et le récit historique. Paris, Seuil.

Saujat, F. 2010. Travail, formation et développement des professionnels de l'éducation : voies de recherche en sciences de l'éducation. Note de synthèse pour l'Habilitation à Diriger des Recherches. Aix en Provence, Université de Provence (Aix-Marseille I).

. 2011. «L'activité enseignante », in Maggi, B. Interpréter l'agir: un défi théorique, Paris, P.U.F.

SEnSEVy, G. 1999. Eléments d'une anthropologie de l'action didactique. Texte présenté pour l'habilitation à diriger les recherches. Aix en Provence, Université de Provence (Aix Marseille I).

Wallon, H. 1942/1970. De l'acte à la pensée. Paris, Flammarion. 